\title{
Postherpetic Pseudohernia: Lesion Localization Using Thoracic Spine Magnetic Resonance Imaging
}

\author{
Jeong-Yoon Lee \\ Jun-Sang Sunwoo \\ Kyum-Yil Kwon \\ Kyung Bok Lee \\ Moo-Young Ahn \\ Hakjae Roh \\ Department of Neurology, \\ Soonchunhyang University \\ School of Medicine, \\ Seoul Hospital, Seoul, Korea
}

Received October 23, 2018

Revised November 25, 2018

Accepted November 28, 2018

\section{Correspondence}

Hakjae Roh, MD, $\mathrm{PhD}$

Department of Neurology,

Soonchunhyang University

School of Medicine, Seoul Hospital,

59 Daesagwan-ro, Yongsan-gu,

Seoul 04401, Korea

Tel +82-2-709-9224

Fax +82-2-795-3687

E-mailnnewroh@schmc.ac.kr
Dear Editor,

Postherpetic pseudohernia is a rare neurological complication of herpes zoster. Electrodiagnostic studies, CT, MRI, and ultrasonography of abdominal wall muscles are helpful for diagnosing postherpetic pseudohernia. ${ }^{1,2}$ Here we present a case of postherpetic pseudohernia showing focal enhancement of the thoracic nerve root and spinal nerve in MRI.

A 56-year-old man came to the outpatient clinic presenting with an abdominal bulge that had appeared 5 days previously. He was suffering from a skin lesion producing stabbing pain on his right flank and severe constipation with had lasted for a month. He had no history of medical illness, physical trauma, smoking habit, or alcohol consumption.

His initial vital signs were blood pressures of $121 / 83 \mathrm{~mm} \mathrm{Hg}$, heart rate of 88 beats/min, respiration rate of 20 breaths $/ \mathrm{min}$, and body temperature of $36.9^{\circ} \mathrm{C}$. A physical examination revealed vesicles and an abdominal bulge without a palpable mass in the right flank following the T10-11 dermatome (Fig. 1A). Abdominal tenderness, rebound tenderness, and auscultation were unremarkable. A neurological examination revealed a decreased right superficial abdominal reflex. Motor and sensory examinations showed no abnormality. Abdominal CT revealed thinning of the right external oblique and internal oblique muscles (Fig. 1B). Focal enhancement of the posterior nerve root and spinal nerve at the T10 vertebral body level was observed in thoracic spine MRI, but no spinal cord lesion was evident (Fig. 1C-F).

Valaciclovir and medications for neuropathic pain were administered. The abdominal bulge had improved 3 months later.

A diagnosis of postherpetic pseudohernia is clinical and can be suspected in a patient with a history of herpes zoster and an abdominal bulge. According to a crucial review of postherpetic pseudohernia, an abdominal bulge commonly appears at 2-6 weeks after the onset of a herpetic rash. A strong correlation has been observed between the dermatomal distribution of the herpetic rash and the abdominal muscle paresis in all reported cases. Visceral neuropathy showing severe constipation, colonic pseudo-obstruction, or neurogenic bladder can occur concomitantly in patients with postherpetic pseudohernia. ${ }^{1}$

The exact pathophysiology of motor neuropathy in herpes zoster is not fully understood. A convincing theory is the direct spread of the varicella zoster virus from dorsal root ganglia to anterior horn cells or anterior spinal nerve. ${ }^{2}$ Lesions of nerve roots from T6 to L1 can induce segmental paresis of the anterolateral abdominal wall muscles, leading to pseudohernia. ${ }^{1}$ Although MRI is helpful for not only localizing and delineating the extent of inflammation in segmental zoster paresis but also excluding other structural abnormalities, ${ }^{3-5}$ there is no previous report of MRI data in postherpetic pseudohernia. ${ }^{1}$ MRI findings in segmental zoster paresis of limbs include lesions of the anterior and posterior spinal nerve roots with abnormal gadolinium enhancement, and lesions of the anterior and posterior horns characterized by high signal intensities on T2-weighted images. ${ }^{3-5}$ Lesions of the T10 posterior nerve root and spinal nerve indicating radiculoneuritis were found in our patient. This finding is

@ This is an Open Access article distributed under the terms of the Creative Commons Attribution Non-Commercial License (https://creativecommons.org/licenses/by-nc/4.0) which permits unrestricted non-commercial use, distribution, and reproduction in any medium, provided the original work is properly cited. 
suggestive of virus-induced inflammation, which is consistent with facial nerve enhancement in Ramsay Hunt syndrome. ${ }^{3}$ Regarding clinicoradiological correlations, lesions of the posterior nerve root and spinal nerve might be associated with neuralgia and pseudohernia, respectively.
Several modalities other than thoracic spine MRI are useful for diagnosing postherpetic pseudohernia. Thinning of abdominal wall muscles that becomes more prominent with standing or Valsalva maneuver can be seen in abdominal CT, abdominal wall MRI, and ultrasonography. ${ }^{6}$ Additionally, an
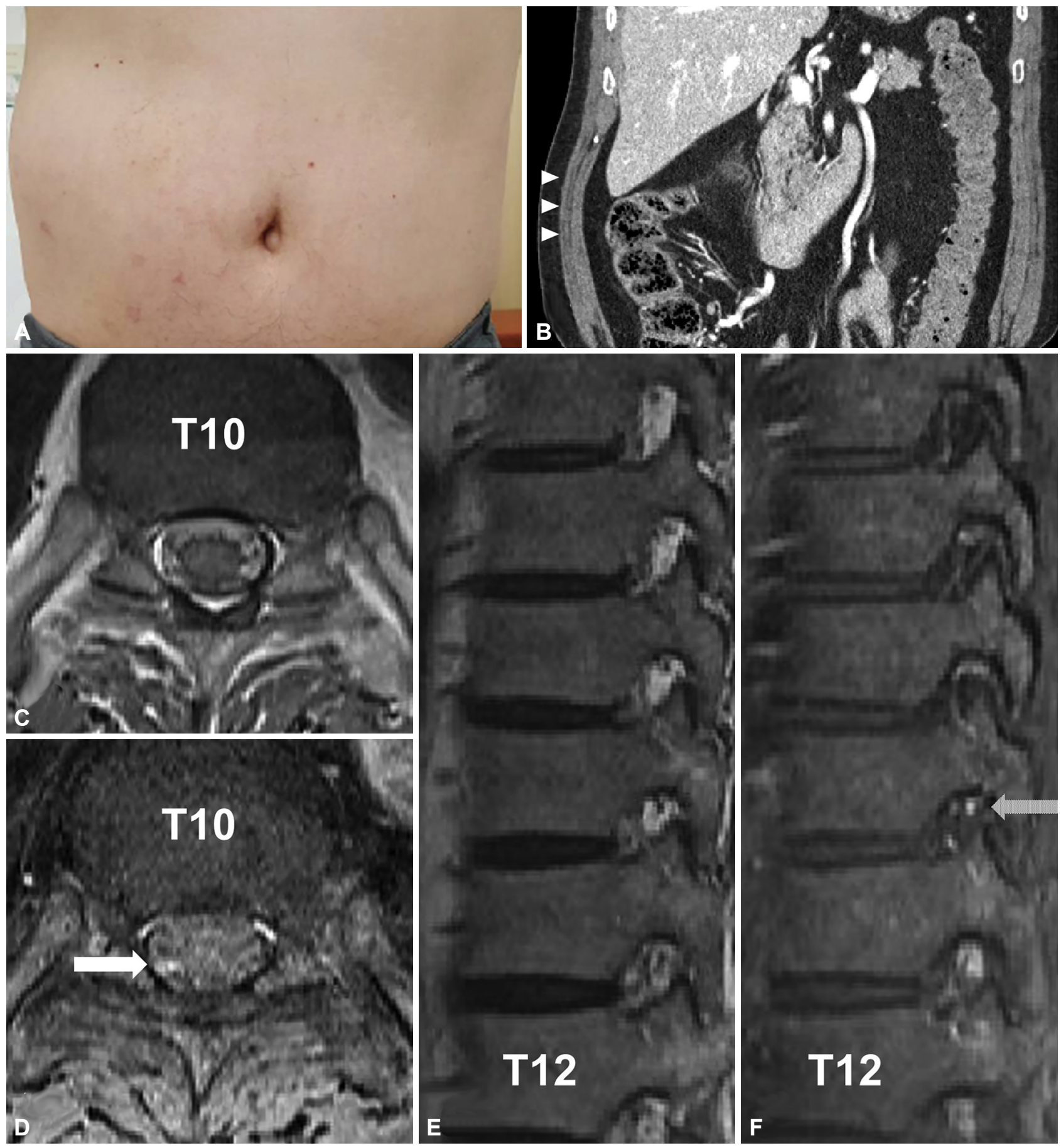

E

Fig. 1. Clinical and radiological findings of the patient. A: Herpetic skin rash and prominent bulging on the right flank were observed. B: Abdominal CT revealed thinning of the right external oblique and internal oblique muscles (arrowheads). C, E: Thoracic spine MRI produced unremarkable T1-weighted images. D, F: Focal enhancement of the posterior nerve root (white arrow) and spinal nerve (gray arrow) at the T10 vertebral body level were found on contrast-enhanced images. 
electrodiagnostic study is a very sensitive tool for demonstrating denervation of the abdominal wall muscles. ${ }^{1}$ An intercostal nerve conduction study and electromyography of the abdominal wall and thoracic paraspinal muscles could be helpful for diagnosing radiculoneuropathy in such patients.

It is important to differentiate postherpetic pseudohernia from an abdominal wall hernia when determining the treatment strategy. Thoracic spine MRI could be useful noninvasive modality for both diagnosing and understanding the pathophysiology by demonstrating inflammation in postherpetic pseudohernia.

\section{Conflicts of Interest}

The authors have no potential conflicts of interest to disclose.

\section{REFERENCES}

1. Chernev I, Dado D. Segmental zoster abdominal paresis (zoster pseudohernia): a review of the literature. $P M \& R$ 2013;5:786-790.

2. Healy C, McGreal G, Lenehan B, McDermott EW, Murphy JJ. Selflimiting abdominal wall herniation and constipation following herpes zoster infection. QJM 1998;91:788-789.

3. Hanakawa T, Hashimoto S, Kawamura J, Nakamura M, Suenaga T, Matsuo M. Magnetic resonance imaging in a patient with segmental zoster paresis. Neurology 1997;49:631-632.

4. Samuraki M, Yoshita M, Yamada M. MRI of segmental zoster paresis. Neurology 2005;64:1138.

5. Umehara T, Sengoku R, Mitsumura H, Mochio S. Neurological picture. Findings of segmental zoster paresis on MRI. J Neurol Neurosurg Psychiatry 2011;82:694.

6. Miranda-Merchak A, García N, Vallejo R, Varela C. MRI findings of postherpetic abdominal wall pseudohernia: a case report. Clin Imaging 2018;50:109-112. 